\title{
Adsorption of symmetric random copolymer onto symmetric random surface: the annealed case
}

\author{
A.A. Polotsky 112 \\ ${ }^{1}$ Institute of Macromolecular Compounds, Russian Academy of Sciences, 31 Bolshoy prospekt, 199004 Saint \\ Petersburg, Russia \\ 2 Saint Petersburg National Research University of Information Technologies, Mechanics and Optics (ITMO \\ University), 49 Kronverkskiy prospekt, 197101 Saint Petersburg, Russia
}

Received September, 24, 2014, in final form February 4, 2015

\begin{abstract}
Adsorption of a symmetric $(A B)$ random copolymer (RC) onto a symmetric $(a b)$ random heterogeneous surface (RS) is studied in the annealed approximation by using a two-dimensional partially directed walk model of the polymer. We show that in the symmetric case, the expected a posteriori compositions of the RC and the RS have correct values (corresponding to their a priori probabilities) and do not change with the temperature, whereas second moments of monomers and sites distributions in the RC and RS change. This indicates that monomers and sites do not interconvert but only rearrange in order to provide better matching between them and, as a result, a stronger adsorption of the RC on the RS. However, any violation of the system symmetry shifts equilibrium towards the major component and/or more favorable contacts and leads to interconversion of monomers and sites.
\end{abstract}

Key words: random copolymer, random surface, polymer adsorption, annealed approximation, generating functions

PACS: 87.10.Hk, 82.35.Gh, 82.35.Jk

\section{Introduction}

Adsorption of heteropolymers - polymers composed of monomer units of two and more types onto chemically heterogeneous surfaces was intensively studied in the last decades. A particular interest to this problem is motivated by its connection to the question of molecular recognition playing a crucial role in living organisms and in various biomedical/biotechnological applications. To understand the mechanisms of the polymer-surface recognition, the problem was extensively investigated from different angles by using relatively simple and physically transparent models.

In particular, directed walk models of polymers [1-3] played an important role in studying homopolymer adsorption [4-12] and collapse [13-17] and related problems of force-induced desorption [18-26] and unfolding of a collapsed macromolecule [27, 28]. Random [29-31] and periodic [32-34] copolymer adsorption and mechanical desorption [35-37] were also studied with the aid of directed models. The advantage of directed models consists in their simplicity; polymer directedness allows one to obtain an exact solution in most cases, typically in the long chain limit. At the same time, they provide a physically reasonable and tractable picture of the phenomenon under study which is commonly in agreement with the results of a more realistic computer simulation of the same (or similar) system in 3 dimensions. Another attractive feature of directed polymer models is the inherent self-avoidance of polymer conformations (and the corresponding impossibility of visiting the same surface site by two different monomer units simultaneously).

To study homo- or heteropolymer adsorption onto a homogeneous surface, one can use fully directed polymer models (in particular, Dyck or Motzkin paths). In the case when both the polymer and the surface are heterogeneous and the polymer should adjust its conformation to the surface pattern in order 
to attain a better matching of its monomer sequence to the heterogeneous surface pattern and maximize the amount of favorable polymer-surface contacts, the minimal model that allows one to consider this phenomenon is the two-dimensional partially directed walk (2D-PDW) model. The fully directed model, which is simpler than the 2D-PDW one, is not suitable for this purpose because for a particular monomer unit, there is one and only one surface site that it can visit (hence, heteropolymer adsorption onto heterogeneous surface is equivalent to the situation of a heteropolymer adsorption onto a homogeneous surface).

In our recent paper [31], a random copolymer (RC) adsorption onto a random surface (RS) was considered in the framework of the 2D-PDW model of the polymer on a square lattice (the "surface", therefore, was simply a line). In order to take correlations into account, both random sequences of monomers (in the RC) and sites (in the RS) were modelled as first-order Markov chains. The problem was solved by using a combination of the annealed approximation to perform double averaging over sequence and surface disorder, and the generating functions (GFs) approach to sum over all conformations of the RC. The key result of the work [31] was the derivation of an equation to find the smallest singularity of the GF of the adsorbed chain. The latter provides an asymptotic form of the canonical partition function for the annealed system which, in turn, gives an access to the calculation of various observables. This equation was then applied in [31] for the analysis of the adsorption transition point for different sets of the system parameters. This allowed us to study the effect of the interplay between correlations in the RC and the RS on the transition temperature.

In the present work, we employ the model introduced in [31] for a comprehensive study of the RC adsorption onto the RS beyond the transition point in the annealed approximation. It is important to note that for the system considered, the annealed approximation is interesting not only as a mathematical trick but due to its correspondence to real physical situations. Mathematically, the annealed approximation is equivalent to direct averaging the partition function of a disordered system instead of averaging its logarithm (i.e., free energy) over all possible realizations of the disorder. For our system this means that monomers and sites participate in thermal motion along with the conformational degrees of freedom, and, following Grosberg [38], we may refer to the annealed RC (RS) as a copolymer (a heterogeneous surface) with a "mobile primary structure".

Yoshinaga et al. [39] developed a theory of adsorption of the so-called two-state polymers consisting of monomer units that can change its type (i.e., its affinity to the substrate or its hydrophobicity/hydrophilicity). The authors of [39] showed the equivalence of the two-state polymer to the annealed two-letter RC and established the correspondence between the RC parameters (a priori probabilities to find a monomer of a certain type in the RC sequence) and standard chemical potentials of monomers in the bulk and at the surface. Another situation that can be described in terms of the annealed approximation was suggested in [38]: the annealed RC can be viewed as a homopolymer consisting of monomer units that can adsorb small molecules, like surfactants [40], from the solution. As a result, there are two types of monomer units: free and with an adsorbed "side group". Correspondingly, monomer units with "side groups" may have additional attraction to each other or to a surface. This picture can be straightforwardly extended to the case where two interacting objects are heterogeneous: both polymer and surface may be "two state" or may bind (different) ligands and, being in a bound state, attract each other.

There exists another aspect that makes the study of RC adsorption onto RS in the annealed approximation interesting. As it is well known, in the case of a RC adsorbing onto a homogeneous surface, the situation develops as follows [29, 41]: with a decreasing temperature, transformation of non-adsorbing monomer units into adsorbing ones occurs. As a result, the a posteriori first and higher moments of the monomer distribution (that is, the number of monomer units of $A$ and $B$ type, the number of dyads: $A A$, $B B, A B$ and $B A$, triads: $A A A, A B A, B A A, \ldots$ and so on) do not correspond to their expectation values. In the case where both interacting objects are heterogeneous and there are two or more possibilities of forming favorable, or "good", contacts (say, there are two kinds of favorable contacts: $A a$ and $B b$ ) it is not easy to predict the system behavior. The most unclear will be the symmetric situation, where both good contacts $(A a$ and $B b$ ) are equally favorable, whereas non-attractive "bad" contacts ( $A b$ and $B a$ ) are equally unfavorable and, in addition, both the RC and the RS have a symmetric composition (i.e., equal amounts of $A$ and $B$ monomers and $a$ and $b$ sites): here, one cannot say in advance how this compositional equilibrium will be biased during the interaction of the RC with the RS upon a decreasing temperature (increasing interaction strength). Phase diagram for the symmetric case was analyzed in [31]: it was shown that RC tends 
to adsorb onto the RS with the same type of correlations: quasi-blocky on quasi-blocky, quasi-alternating on quasi-alternating whereas uncorrelated, or Bernoullian, RCs (RSs) do not "feel" correlations on the RS (in the RC). These findings are in line with the results of Monte Carlo simulations [42] for a more realistic three-dimensional self-avoiding chain.

The rest of the paper is organized as follows. In section 2 the model and the formalism based on the annealed approximation and the GFs approach are introduced. Presentation of the results is preceded by section 3 where a simpler system-a RC adsorbing onto a homogeneous surface-is considered to illustrate the main features of the annealed approximation. This system also serves us as a reference. Section 4 is the main part of the paper devoted to the exposition of the results in the case of symmetric composition and symmetric "interaction map" ("interaction matrix"). We present temperature dependences of the total and partial adsorbed fractions, analyze the effect of a variation in the composition and in the character of correlations in the RC and the RS, discuss the effect of asymmetry in the composition and/or interaction matrix on the run of these dependences. Finally, we summarize in section 5

\section{Model and method}

\subsection{Definition of the model}

Consider a RC chain composed of $A$ and $B$ monomer units interacting with a RS that carries $a$ and $b$ sites, figure 1 (note that the two-species heteropolymer chain is similar to the so-called hydrophobicpolar (HP) copolymer, a model widely used in theoretical studies of protein folding [43, 44]). As in [31], we model polymer conformations by 2D-PDWs on the square lattice, therefore, the adsorbing surface is simply a line. The monomer sequence of the RC is given by $\chi=\left\{\chi_{1}, \chi_{2}, \ldots, \chi_{n}\right\}$, where $\chi_{i}=A$ or $B$ while the surface pattern is given by $\sigma=\left\{\ldots, \sigma_{1}, \sigma_{2}, \sigma_{3}, \ldots\right\}$, where $\sigma_{x}=a$ or $b$.

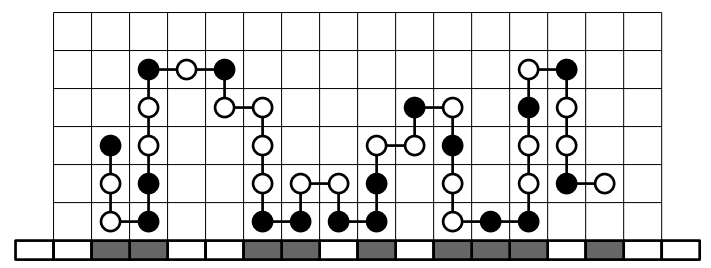

Figure 1. 2D partially directed walk model of random copolymer consisting of $A$ (black) and $B$ (white) monomer units near the linear ("planar") random surface composed of $a$ (gray) and $b$ (white) sites.

Monomer units and surface sites are distributed randomly and modelled as the first order Markov chain. For the RC, the latter is determined by the probabilities to find $A$ and $B$ units in the sequence: $P\left(\chi_{i}=A\right)=f_{A}$ and $P\left(\chi_{i}=B\right)=f_{B}=1-f_{A}$, and by the probabilities that the monomer of the type $i$ is followed by the monomer of the type $j, P\left(\chi_{m}=j \mid \chi_{m-1}=i\right)=p_{i j}$. The correlation parameter $c_{\mathrm{p}}=$ $1-p_{A B}-p_{B A}=p_{A A}+p_{B B}-1$ determines the character of correlations in the sequence: $c_{\mathrm{p}}>0$ means that there is a tendency in the sequence for grouping similar monomers into clusters, $c_{\mathrm{p}}<0$ favors the alternating sequence of $A$ 's and $B$ 's, $c_{\mathrm{p}}=0$ corresponds to uncorrelated (Bernoullian) sequences. The probability of occurrence of a particular realization of the sequence $\chi$ is given by the product $P(\chi)=$ $f_{\chi_{1}} p_{\chi_{1} \chi_{2}} p_{\chi_{2} \chi_{3}} \cdot \ldots \cdot p_{\chi_{n-1} \chi_{n}}$.

Similarly, the sequence of surface sites is determined by the probabilities to find $a$ and $b$ site on the surface $P\left(\sigma_{x}=a\right)=g_{\mathrm{a}}$ and $P\left(\sigma_{x}=b\right)=g_{b}=1-g_{\mathrm{a}}$ and the probabilities that the site of the type $i$ is followed by the site of the type $j, P\left(\sigma_{x}=j \mid \sigma_{x-1}=i\right)=s_{i j}$. The correlation parameter for the surface $c_{\mathrm{S}}=$ $1-s_{a b}-s_{b a}=s_{a a}+s_{b b}-1$. The probability of a particular realization of the sequence $\sigma=\left\{\sigma_{1}, \sigma_{2}, \ldots, \sigma_{\ell}\right\}$ is given by the product $P(\sigma)=g_{\sigma_{1}} s_{\sigma_{1} \sigma_{2}} s_{\sigma_{2} \sigma_{3}} \cdot \ldots \cdot s_{\sigma_{\ell-1} \sigma_{\ell}}$.

The Hamiltonian (the energy) of the system can be written as follows:

$$
H=\sum_{i=1}^{N} \delta_{y_{i}, 0} \epsilon_{\chi_{i} \sigma_{x_{i}}},
$$


and the partition function of the system for particular realizations of the RC and the RS is given by

$$
Z_{n}(\beta \mid \chi, \sigma)=\sum_{\mathbf{r}_{i}} \exp \left[-\beta \sum_{i=1}^{N} \delta_{y_{i}, 0} \epsilon_{\chi_{i} \sigma_{x_{i}}}\right],
$$

where $\mathbf{r}_{i}=\left\{x_{i}, y_{i}\right\}, i=1, \ldots, n$ denotes the chain conformation (the trajectory of the chain), $\delta_{i j}$ is the Kronecker delta, $\epsilon_{i j}$ is the energy of the monomer-surface contact ( $i \in\{A, B\}$ and $j \in\{a, b\}$ ), and $\beta=$ $1 / k_{\mathrm{B}} T$ is the inverse temperature.

\subsection{Annealed approximation and generating functions approach}

We will solve the problem in the annealed approximation, where the quenched free energy $\beta F_{\mathrm{q}}=$ $-\left\langle\left\langle\ln Z_{n}(\beta \mid \chi, \sigma)\right\rangle_{\chi}\right\rangle_{\sigma}$ obtained by averaging the logarithm of the partition function over all possible realizations of the RC and the RS is approximated by the annealed free energy $\beta F_{\mathrm{a}}=-\ln \left\langle\left\langle Z_{n}(\beta \mid \chi, \sigma)\right\rangle_{\chi}\right\rangle_{\sigma}$, where the partition function is averaged prior to taking the logarithm. Here, the angular brackets $\langle\ldots\rangle$ denote averaging over sequence or surface randomness.

To calculate the annealed partition function of the system considered, $\left\langle\left\langle Z_{n}(\beta \mid \chi, \sigma)\right\rangle_{\chi}\right\rangle_{\sigma}$, we use the generating functions (GFs) approach (or the grand canonical approach). In the case of adsorption onto heterogeneous surface, this approach consists in calculating the GF

$$
\Xi(z, t)=\sum_{n=1}^{\infty} \sum_{m=1}^{n}\left\langle\left\langle Z_{n, m}(\beta \mid \chi, \sigma)\right\rangle_{\chi}\right\rangle_{\sigma} z^{n} t^{m},
$$

where $Z_{n, m}(\beta \mid \chi, \sigma)$ is the constrained partition function of a chain with $n$ monomer units and the length of the chain projection onto adsorbing substrate equal to $m$. The GF variables $z$ and $t$ conjugate to the chain length and the chain projection, respectively. The smallest singularity $z_{c}(t)$ of $\Xi(z, t)$ calculated at $t=1$ gives an asymptotic expression for the partition function in the large $n$ limit: $\left\langle\left\langle Z_{n}(\beta \mid \chi, \sigma)\right\rangle_{\chi}\right\rangle_{\sigma} \simeq$ $z_{\mathrm{c}}^{-n}(t=1)$. Then, the monomer chemical potential (the free energy per monomer unit) $\mu=\ln \left[z_{\mathrm{c}}(t=1)\right]$

As it was shown in [31], the smallest singularity of $\Xi(z, t)$ is associated with the smallest root of the equation

$$
\operatorname{det}\left[\mathbf{E}-\Xi_{\mathrm{L}}(z, t) \Xi_{\mathrm{S}}(z t)\right]=0
$$

In equation (2.4), the functions $\Xi_{\mathrm{S}}(z t), \Xi_{\mathrm{L}}(z, t)$ are the GFs of adsorbed segments (usually called "trains”) and loops, respectively, in the matrix form:

$$
\begin{aligned}
& \Xi_{\mathrm{S}}(z t)=\sum_{n=1}^{\infty} \Omega_{\mathrm{S}}(n)(z t)^{n} \mathbf{R}^{n}, \\
& \Xi_{\mathrm{L}}(z, t)=\sum_{n=2}^{\infty} \sum_{m=2}^{n} \Omega_{\mathrm{L}}(n, m) z^{n} t^{m-2}\left(\mathbf{P}^{n} \otimes \mathbf{S}^{m-2}\right),
\end{aligned}
$$

where $\Omega_{\mathrm{L}}(n, m)$ is the number of loops of contour length $n$ and projection length $m$ and $\Omega_{\mathrm{S}}(n, m)$ is the number of trains of length $n$. The matrices $\mathbf{P}$ and $\mathbf{S}$ are the transition probability matrices for RC and RS, respectively:

$$
\begin{aligned}
& \mathbf{P}=\left(\begin{array}{ll}
p_{A A} & p_{A B} \\
p_{B A} & p_{B B}
\end{array}\right)=\left(\begin{array}{cc}
p_{A A} & 1-p_{A A} \\
1-p_{B B} & p_{B B}
\end{array}\right), \\
& \mathbf{S}=\left(\begin{array}{ll}
s_{a a} & s_{a b} \\
s_{b a} & s_{b b}
\end{array}\right)=\left(\begin{array}{cc}
s_{a a} & 1-s_{a a} \\
1-s_{b b} & s_{b b}
\end{array}\right),
\end{aligned}
$$

$\mathbf{P} \otimes \mathbf{S}$ is the Kronecker product of the these matrices. The matrix $\mathbf{R}$ is defined as

$$
\mathbf{R}=(\mathbf{P} \otimes \mathbf{S}) \cdot \mathbf{W}
$$

with the diagonal "interaction matrix"

$$
\mathbf{W}=\operatorname{diag}\left(w_{A a}, w_{A b}, w_{B a}, w_{B b}\right)
$$


containing all statistical weights of different monomer-surface contacts, $w_{i j} \equiv \mathrm{e}^{-\beta \epsilon_{i j}}$, where $i \in\{A, B\}$ and $j \in\{a, b\}$.

The matrix GFs $\Xi_{\mathrm{S}}(z t)$ and $\Xi_{\mathrm{L}}(z, t)$ can be easily calculated by using the eigenvalues and eigenvectors of the matrices $\mathbf{P}, \mathbf{S}$, and $\mathbf{R}$ and the expressions for scalar GFs of trains calculated straightforwardly as follows:

$$
\Xi_{\mathrm{S}}(z)=\sum_{n=1}^{\infty} \Omega_{\mathrm{S}}(n) z^{n}=z^{2}+z^{3}+\cdots=\frac{z^{2}}{1-z},
$$

and loops (calculated by using a loop decomposition described in [31])

$$
\Xi_{\mathrm{L}}(z, t)=\sum_{n=2}^{\infty} \sum_{m=2}^{n} \Omega_{\mathrm{L}}(n, m) z^{n} t^{m-2}=\frac{1-z t-z^{2}-z^{3} t-\sqrt{\left(1-z t-z^{2}-z^{3} t\right)^{2}-4 z^{4} t^{2}}}{2 z^{2} t^{2}},
$$

see also [31], equations (23)-(25) for details.

Note that the determinant equation (2.4) is a generalization of the analogous scalar equation for a homopolymer adsorption onto a homogeneous surface [45]: $1-\Xi_{\mathrm{L}}(z) \Xi_{\mathrm{S}}(w z)=0$, where $w=\mathrm{e}^{-\beta \epsilon}$ is the statistical weight of a monomer-surface contact, $\Xi_{\mathrm{L}}(z)=\Xi_{\mathrm{L}}(z, 1)$.

The smallest singularity $z_{\mathrm{C}}$ must then be compared with the smallest singularity $z_{\mathrm{V}}$ of the GF for the free (desorbed) chain in a bulk $\Xi_{\mathrm{V}}(z)=\sum_{n=1}^{\infty} \Omega_{\mathrm{V}}(n) z^{n}$, which does not depend on monomer sequence. Here, $\Omega_{\mathrm{V}}(n)$ is the number of conformation that a chain of $n$ monomer units can acquire in the bulk. For the 2D-PDW polymer model $z_{\mathrm{V}}=\sqrt{2}-1[1]$.

In the adsorption transition point $z_{\mathrm{c}}=z_{\mathrm{V}}$, hence, the equation

$$
\operatorname{det}\left[\mathbf{E}-\Xi_{\mathrm{L}}\left(z_{\mathrm{V}}, t\right) \Xi_{\mathrm{S}}\left(z_{\mathrm{V}} t\right)\right]_{t=1}=0
$$

determines the position of the transition point.

\subsection{Calculation of observables}

Various observables can be found via differentiation of the smallest singularity $z_{\mathrm{c}}(t)$ of the GF. For example, logarithmic derivative of $z_{\mathrm{c}}$ with respect to the statistical weight of different monomer-surface contacts, $w_{i j}$, gives the average fraction of these contacts occurring in the adsorbed RC chain:

$$
\theta_{i j}=-\frac{\partial \ln z_{\mathrm{c}}}{\partial \ln w_{i j}}=-\frac{w_{i j}}{z_{\mathrm{c}}} \cdot \frac{\partial z_{\mathrm{c}}}{\partial w_{i j}} .
$$

The total adsorbed fraction is given by the sum of four contributions: $\theta=\theta_{A a}+\theta_{A b}+\theta_{B a}+\theta_{B b}$.

The partial derivative $\partial z_{\mathrm{c}} / \partial w_{i j}$ in equation 2.12 can be calculated by differentiating the equation (2.4). Namely, if we denote in the left-hand side of equation (2.4) as $D:=\operatorname{det}\left[\mathbf{E}-\Xi_{\mathrm{L}}(z, t) \Xi_{\mathrm{S}}(z t)\right]$, then

$$
\frac{\partial z_{\mathrm{c}}}{\partial w_{i j}}=-\left.\frac{\partial D / \partial w_{i j}}{\partial D / \partial z}\right|_{z=z_{\mathrm{c}}, t=1}
$$

The derivatives of $D$ can be found with the aid of Jacobi's formula

$$
\frac{\mathrm{d} \operatorname{det} \mathbf{X}(\tau)}{\mathrm{d} \tau}=\operatorname{Tr}\left(\operatorname{Adj}(\mathbf{X}) \cdot \frac{\mathrm{d} \mathbf{X}}{\mathrm{d} \tau}\right)
$$

where "Tr" stands for the trace of a matrix and $\operatorname{Adj}(\mathbf{X})$ denotes the adjugate matrix for $\mathbf{X}$.

By analogy, one can calculate other observables by choosing a proper differentiation variable. Taking a derivative of $z_{\mathrm{c}}(t)$ with respect to $t$ gives access to the ratio of the average projection of the RC chain onto the surface to the RC contour length:

$$
\frac{\langle m\rangle}{n}=-\left.\frac{\partial \ln z_{\mathrm{c}}}{\partial \ln t}\right|_{t=1}=-\left.\frac{1}{z_{\mathrm{c}}} \cdot \frac{\partial z_{\mathrm{c}}}{\partial t}\right|_{t=1} .
$$

Since in the annealed system, RC monomer units and RS sites are in thermal motion, it is especially interesting to find equilibrium moments of their distributions. To calculate the fraction of $A$ monomers 
in the RC chain, $v_{A}$, let us introduce an auxiliary term into the Hamiltonian (2.1): we set $H \rightarrow H+\Delta H$, where

$$
\Delta H=-(\eta / \beta) \sum_{i=1}^{N} \chi_{i}
$$

Later $\eta$ will be set to zero. With this auxiliary term, the matrix $\mathbf{P}$ modifies as follows:

$$
\mathbf{P}=\left(\begin{array}{cc}
p_{A A} \mathrm{e}^{\eta} & p_{A B} \\
p_{B A} \mathrm{e}^{\eta} & p_{B B}
\end{array}\right)
$$

The matrix $\mathbf{R}$ is again $\mathbf{R}=(\mathbf{P} \otimes \mathbf{S}) \cdot \mathbf{W}$. Then, the average fraction of $A$ monomers in the $\mathrm{RC}$ chain is given by

$$
v_{A}=-\left.\frac{\partial \ln z_{\mathrm{c}}}{\partial \eta}\right|_{\eta=0}=-\left.\frac{1}{z_{\mathrm{c}}} \cdot \frac{\partial z_{\mathrm{c}}}{\partial \eta}\right|_{\eta=0} .
$$

The fraction of $B$ units follows automatically: $v_{B}=1-v_{A}$.

Similarly, one can calculate the average fraction of $A A, A B, B A$, or $B B$ dyads in the sequence. For example, $v_{A A}$ is calculated by introducing the following auxiliary term:

$$
\Delta H=-(\eta / \beta) \sum_{i=2}^{N} \chi_{i-1} \chi_{i}
$$

This modifies the matrix $\mathbf{P}$ in the following way:

$$
\mathbf{P}=\left(\begin{array}{cc}
p_{A A} \mathrm{e}^{\eta} & p_{A B} \\
p_{B A} & p_{B B}
\end{array}\right),
$$

i.e., the multiplier $\mathrm{e}^{\eta}$ appears at the corresponding element ( $\left.p_{A A}\right)$ of the probability matrix. The cluster parameter $\lambda_{\mathrm{p}}$, which is the a posteriori analogue of the a priori correlation parameter $c_{\mathrm{p}}$, is calculated as $\lambda_{\mathrm{p}}=1-v_{A B} / v_{A}-v_{B A} / v_{B}=v_{A A} / v_{A}-v_{B B} / v_{B}-1$.

In a similar manner, we can use the same idea to obtain the composition of RS. Thus, to calculate the fraction of $a$ sites on the surface, the matrix $\mathbf{S}$ should be modified as follows:

$$
\mathbf{S}=\left(\begin{array}{cc}
s_{a a} \mathrm{e}^{\eta} & s_{a b} \\
s_{b a} \mathrm{e}^{\eta} & s_{b b}
\end{array}\right)
$$

However, there is an important difference between RC and RS: while in the former case all monomer units can be involved in the interaction with the surface, in the latter case only a part of the RS may be involved in the interaction with the polymer chain, the size of this "contact zone" is equal to the projection of the RC on the substrate $m \leqslant n$. Mathematically, this difference is expressed in the limits of summation in the GF of equation (2.3). In this sense, the quantity "average fraction of $a$ sites on the surface" is not well defined for the whole surface because if one takes a finite but large surface which cannot be completely covered by the RC there will be two parts of the surface, i.e., a part involved and a part not involved into interaction with the RC. A better quantity is the "local" fraction of $a$-sites, i.e., the ratio of the number of $a$ sites in the "contact zone", $m_{\mathrm{a}}$ to the (instantaneous) size of this zone $m$.

An expression analogous to equation (2.18) does not give the sought fraction of $a$ sites on the surface. It gives the ratio of the average number of $a$ sites on the surface "occupied" by the RC, $m_{\mathrm{a}}$, to the contour length i.e. $m_{\mathrm{a}} / n$. Therefore, a correct estimate of the "local" fraction of $a$-sites, $v_{\mathrm{a}}$, will be given by

$$
v_{\mathrm{a}}=-\left.\frac{\partial \ln z_{\mathrm{c}}}{\partial \eta}\right|_{\eta=0}\left(\frac{\langle m\rangle}{n}\right)^{-1}=-\left.\frac{1}{z_{\mathrm{c}}} \cdot \frac{\partial z_{\mathrm{c}}}{\partial \eta}\right|_{\eta=0}\left(\frac{\langle m\rangle}{n}\right)^{-1}
$$

and similarly for the number of dyads. The cluster parameter for the RS is calculated as $\lambda_{\mathrm{s}}=1-v_{a b} / v_{\mathrm{a}}-$ $v_{b a} / v_{b}$. 


\section{Reference system: RC adsorbed on homogeneous substrate}

In the Introduction we briefly discussed the merits and demerits and the fields of application of the annealed approximation in the study of (various) disordered systems. It will be instructive to illustrate some peculiarities of the annealed approximation in the case of a simpler system, where a RC is adsorbed onto a homogeneous surface. This problem was studied earlier: while in [29], the Bernoullian RC was considered, in [41] a general program for correlated Markovian RC in the framework of the GF approach on the lattice was developed. The results in [41] were obtained for a simple random walk model of polymer in three dimensions, although the approach is quite general and may be straightforwardly extended to other types of lattice models of polymers or to other geometries of adsorbing substrates. In the present work, we apply this program for the 2D-PDW model used in the present study. This will also serve as a "reference system" for comparison with our "original" system.

As it was shown [41], for a RC adsorbed onto a strictly homogeneous surface, the smallest singularity of the GF $\Xi(z)=\sum_{n=1}^{\infty}\left\langle Z_{n}(\beta \mid \chi)\right\rangle_{\chi} z^{n}$ in the annealed approximation is found from the equation similar to equation [2.4): $\operatorname{det}\left[\mathbf{E}-\Xi_{\mathrm{L}}(z) \Xi_{\mathrm{S}}(z)\right]=0$, with matrix GFs $\Xi_{\mathrm{L}}(z)=\sum_{n=2}^{\infty} \Omega_{\mathrm{L}}(n) z^{n} \mathbf{P}^{n}$ and $\Xi_{\mathrm{S}}(z)=$ $\sum_{n=2}^{\infty} \Omega_{\mathrm{S}}(n) z^{n} \mathbf{R}^{n}, \mathbf{R}=\mathbf{P} \cdot \mathbf{W}$, where $\mathbf{W}=\operatorname{diag}\left(w_{A}, w_{B}\right)=\operatorname{diag}\left(\mathrm{e}^{-\beta \epsilon_{A}}, \mathrm{e}^{-\beta \epsilon_{B}}\right)$ is the diagonal matrix of statistical weights of $A$ and $B$ contacts with the surface. Calculation of observables is similar to that described is section 2.3. For more details see [41].

These formulas can also be directly obtained from equations $2.3-(2.8$ by assuming that the RS contains only $a$ sites and, correspondingly, by replacing the matrix $\mathbf{S}$ in equations $2.3-2.7$ by $1 \times 1$ unity matrix, $\mathbf{S}=1$, redefining $\epsilon_{A a} \equiv \epsilon_{A}$ and $\epsilon_{B a} \equiv \epsilon_{B}$, and setting $t=1$. Then, the interaction matrices $\mathbf{W}$, equation (2.8), and $\mathbf{R}$, equation (2.7) will have the dimensionality $2 \times 2$. Alternatively, one can consider $a$ and $b$ sites as identical, i.e., set $\epsilon_{A a}=\epsilon_{A b}=\epsilon_{A}$ and $\epsilon_{B a}=\epsilon_{B b}=\epsilon_{B}$ and then directly use the formalism introduced in section 2

We consider the case of adsorbing $A$ and neutral $B$ contacts: $\epsilon_{A}=-1, \epsilon_{B}=0$. Without loss of generality, let us assume that $A$ and $B$ monomers have equal probabilities to be found in the monomer sequence, $f_{A}=f_{B}=0.5$ but have various character of correlations in the RC chain (i.e., various $c_{\mathrm{p}}$ ). For the sake of comparison, we also consider the homopolymer (HP) consisting of adsorbing $A$ monomer units only.

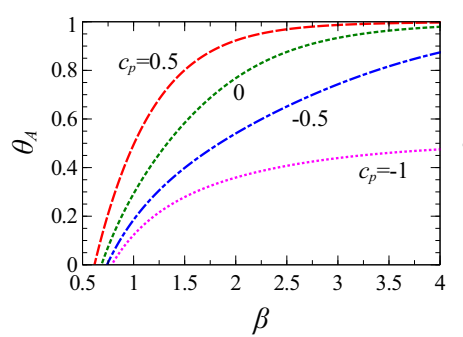

(a)

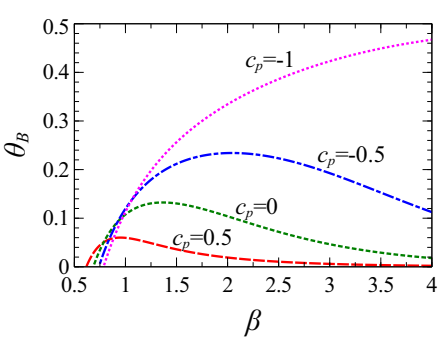

(b)

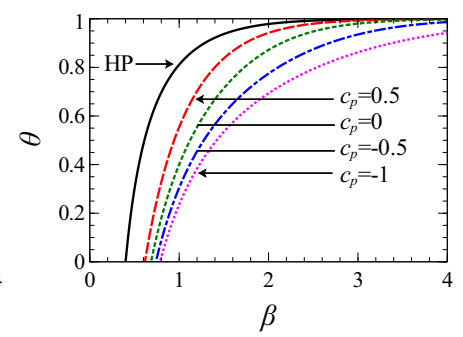

(c)

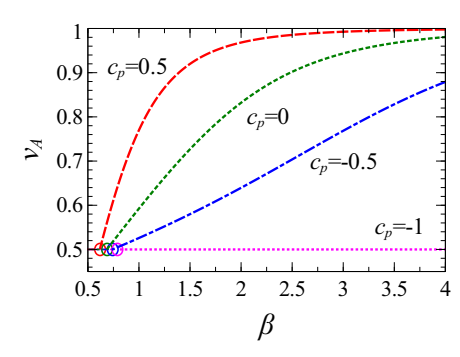

(d)

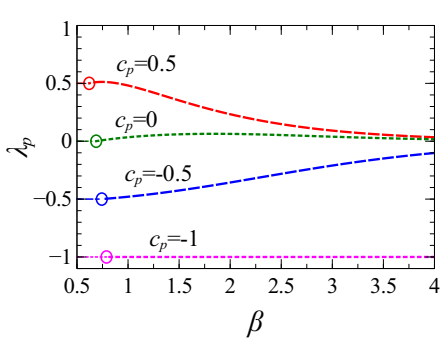

(e)

Figure 2. (Color online) Fraction of $A$ - (good) (a) and $B$ - (bad) (b) contacts, total adsorbed fraction (c), average fraction of $A$-monomers, and (d) cluster parameter for RC (e) in the reference system as functions of inverse temperature $\beta$ calculated for $f_{A}=0.5$ and various $c_{\mathrm{p}}$ as indicated. 
Figure 2 shows temperature dependences of contact fractions (a, b), total adsorbed amount (c), fraction of $A$ monomers $v_{A}(\mathrm{~d})$, and cluster parameter $\lambda_{\mathrm{p}}(\mathrm{e})$. At $c_{\mathrm{p}}>-1$, with an increasing $\beta$, the number of adsorbed $A$ monomers as well as the overall adsorbed fraction monotonously increase while the fraction of adsorbed $B$ units behaves non-monotonously and vanishes at very high $\beta$. This is accompanied by interconversion of repelling monomer units $B$ into adsorbing units $A$ (however, at $\beta \leqslant \beta_{\operatorname{tr}}$, where $\beta_{\text {tr }}$ is the transition point, $v_{A}=f_{A}$ and $\lambda_{\mathrm{p}}=c_{\mathrm{p}}$ ) which explains the observed depencences of $\theta_{A}$ and $\theta_{B}$. Only in the case $c_{\mathrm{p}}=-1$, corresponding to the regularly alternating $A B$-copolymer its regular (i.e., quenched) sequence does not change and both $\theta_{A}$ and $\theta_{B}$ grow with increasing $\beta$ due to a cooperative effect. Therefore, the annealed approximation corresponds to the physical situation that essentially differs from the quenched case.

\section{Results and discussion}

\subsection{Choosing the system parameters}

The system we consider depends on a large number of variables (to be precise, there are 9 parameters: $f_{A}, g_{\mathrm{a}}, c_{\mathrm{p}}, c_{\mathrm{s}}, \epsilon_{A a}, \epsilon_{A b}, \epsilon_{B a}, \epsilon_{B b}$, and $\beta$ ). In [31] we have suggested a reasonable choice of parameters by keeping the inverse temperature $\beta=1 / k_{\mathrm{B}} T$ as a separate control variable and by fixing attractive and repulsive energies, thus obtaining physically relevant temperature dependences. In [31], three different "interaction schemes" for the monomer-site interaction energies were considered, the choice was also motivated by the works of other authors.

Since the present work is devoted to the study of a particular case of the RC and RS symmetric with respect both to composition, $f_{A}=g_{\mathrm{a}}=0.5$, and monomer-site interactions, $\epsilon_{A a}=\epsilon_{B b}$ and $\epsilon_{A b}=\epsilon_{B a}$, this restricts even more the set of variable parameters; in fact, there remain only three ones: $c_{\mathrm{p}}, c_{\mathrm{s}}$, and $\beta$. Therefore, the main part of the present work will be devoted to the study of the symmetric (but highly non-trivial) case; the effect of the asymmetry on the RC and the RS adsorption behaviour will be briefly discussed in the end of this section. We will study the system with the following set of parameters: $A a$ and $B b$ contacts are favorable, $\epsilon_{A a}=\epsilon_{B b}=-1, A b$ and $B a$ contacts are neutral, $\epsilon_{A b}=\epsilon_{B a}=0$.

\subsection{Phase diagram and symmetry properties}

In [31], phase diagram, i.e., the dependence of the inverse adsorption transition temperature $\beta_{\mathrm{tr}}$ on the correlation parameters $c_{\mathrm{p}}$ and $c_{\mathrm{s}}$, was calculated. Figure 3 shows this diagram as a density plot in $\left(c_{\mathrm{p}}, c_{\mathrm{s}}\right)$ coordinates (in such a form it was not presented in [31]). As it follows from the diagram, the smallest values of $\beta_{\text {tr }}$ (dark color, the bottom of the color scale in figure 3) are observed in the vicinity of $\left(c_{\mathrm{p}}, c_{\mathrm{s}}\right) \approx(1,1)$ and $(-1,-1)$ whereas the largest values of $\beta_{\text {tr }}$ (light color, the top of the color scale in figure 3 are observed in the vicinity of $\left(c_{\mathrm{p}}, c_{\mathrm{s}}\right) \approx(-1,1)$ and $(1,-1)$. We can also observe that this density plot is symmetric with respect to the origin $\left(c_{\mathrm{p}}, c_{\mathrm{s}}\right)=(0,0)$. This means that $\beta_{\operatorname{tr}}\left(c_{\mathrm{p}}=x, c_{\mathrm{s}}=y\right)=\beta_{\mathrm{tr}}\left(c_{\mathrm{p}}=-x, c_{\mathrm{s}}=\right.$ $-y$ ) where $x$ and $y$ may take on any value in the interval $(-1,1)$. This symmetry of the diagram is the consequence of the interaction and composition symmetries. (At a glance it may also seem that the diagram is also symmetric with respect to the diagonals $c_{\mathrm{s}}= \pm c_{\mathrm{p}}$ but this is not the case).

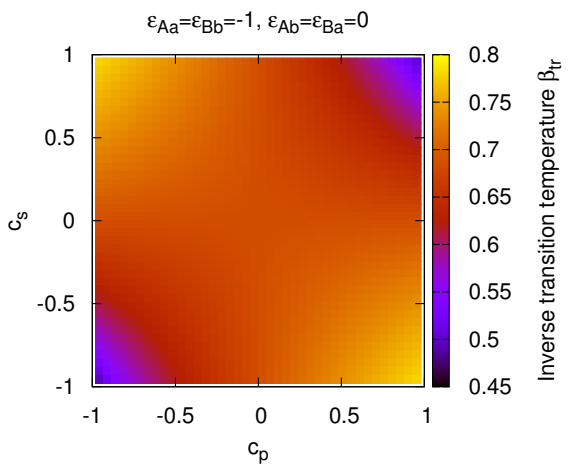

Figure 3. (Color online) Inverse transition temperature in the annealed approximation as function of RC and RS correlation parameters for $\epsilon_{A b}=\epsilon_{B a}=$ $-1, \epsilon_{A b}=\epsilon_{B a}=0$ in the symmetric case $f_{A}=g_{\mathrm{a}}=$ 0.5 . 
This symmetry manifests itself in temperature dependences of the observables beyond the transition point. We vary the values for the correlation parameter for the RC and the RS considering the cases $c_{\mathrm{p}}=-0.5,0,0.5$, and $c_{\mathrm{s}}=-1,-0.5,0,0.5$. It is clear that various combinations of $c_{\mathrm{p}}$ and $c_{\mathrm{s}}$ are possible ( $3 \times 4=12$ combinations). However, due to the interaction and the composition symmetries, it turns out that these 12 dependences for an adsorbed fraction can be presented on the same plot by 5 curves.

\subsection{Adsorbed fraction}

Figure 4 (a)-(c) shows the dependences of the fraction of good $(A a, B b)$ and bad $(A b, B a)$ contacts and of the total adsorbed fraction, respectively, on the inverse temperature $\beta$. Due to the symmetry of the system, we have $\theta_{A a}=\theta_{B b}$ and $\theta_{A b}=\theta_{B a}$. With increasing $\beta$, the fraction of good contacts grows, the maximum "saturation" value for both $A a$ and $B b$ contacts equals 0.5, which is the maximum that is available a priori (according to the values of $f_{A}$ and $g_{\mathrm{a}}$ ), i.e., that $A(B)$ monomer units and $a(b)$ surface sites may form. For the total amount of good contacts $\theta_{\text {good }}=\theta_{A a}+\theta_{B b}$, the upper boundary is equal to unity. In other words, this indicates that $A \rightleftharpoons B$ and $a \rightleftharpoons b$ transformations do not occur, as opposed to the reference system where the fraction of good $A$-contacts grows as $\beta$ increases, figure 2(a). The fraction of bad contacts, figure 2 (b), behaves non-monotonously: just above the adsorption transition it grows with an increasing $\beta$ but then decays to zero. Since the fraction of bad contacts is much lower than the fraction of good contacts, the overall adsorbed fraction increases monotonously with $\beta$, figure 4 (c), and the energy per monomer unit, figure 4 (d), monotonously decreases from 0 to -1 . All the curves start in

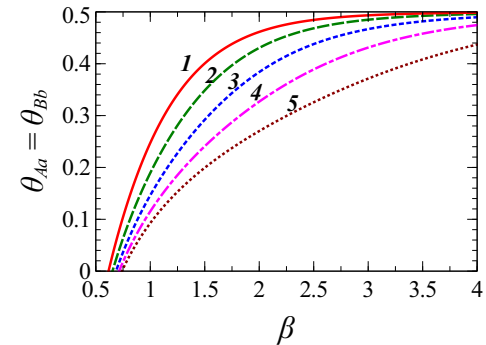

(a)

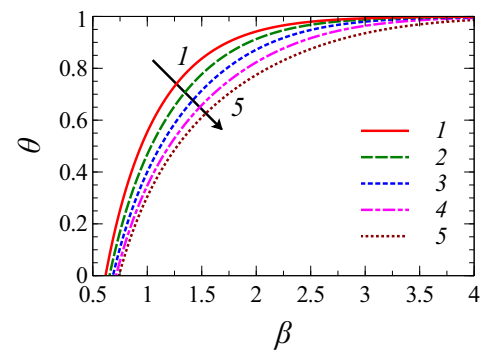

(c)

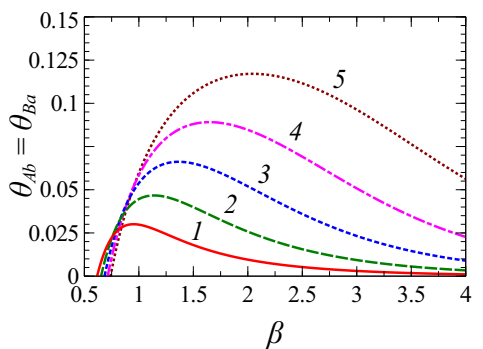

(b)

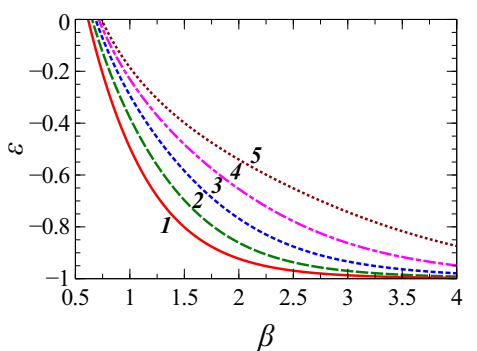

(d)

Figure 4. (Color online) Fraction of good (a) and bad (b) contacts, total adsorbed fraction (c), and energy (d) as functions of inverse temperature $\beta$ calculated for $f_{A}=g_{\mathrm{a}}=0.5$, and various values of cluster parameters-curve 1: $c_{\mathrm{p}}=-0.5, c_{\mathrm{s}}=-1$; curve $2: c_{\mathrm{p}}= \pm 0.5, c_{\mathrm{s}}= \pm 0.5$; curve $3: c_{\mathrm{p}}=0$ or/and $c_{\mathrm{s}}=0$; curve 4: $c_{\mathrm{p}}= \pm 0.5, c_{\mathrm{S}}=\mp 0.5$; curve 5: $c_{\mathrm{p}}=0.5, c_{\mathrm{s}}=-1$.

the transition points ranged in accordance with the phase diagram, figure 3

\subsection{Moments of monomers' and sites' distributions}

In order to understand the behavior of the observables (which is, as we see, much more reasonable and closer to what one can expect in the quenched system, as compared to the reference system) bet- 
ter, we study the temperature dependences of a posteriori moments of distributions of monomers and sites. The first remarkable result is as follows: in the considered symmetric case, the fractions of $A(B)$ monomers and $a(b)$ sites do not depend on the temperature and are always equal to 0.5 ! This takes place at any combinations of correlation parameters $c_{\mathrm{p}}$ and $c_{\mathrm{s}}$ (therefore, we do not show these dependences in the figure due to their trivial form).

As regards the second moments of the a posteriori distributions, these are changing with $\beta$. This means that there are transformations in the RC and the RS sequences but since the RC and the RS compositions are invariant, the transformations occur according to a specific law: $A$ and $B$ units ( $a$ and $b$ sites) move in the RC (RS) in order to tune their sequences with respect to each other in the best way to reach the lowest interaction energy. Alternatively, these rearrangements can be considered as coupled chemical reactions: for example, a transformation $\left(\chi_{i}=A\right) \rightarrow\left(\chi_{i}=B\right)$ should occur simultaneously with the reaction $\left(\chi_{j}=B\right) \rightarrow\left(\chi_{j}=A\right), i$ and $j \neq i$ denote positions of the monomer unit in the RC sequence. At the same time, another important rule sill holds: in the transition point, the first and the second moments of monomers and sites distributions are equal to the corresponding a priori probabilities.

Now, let us consider in detail some particular cases of RC adsorption onto RS.

\subsubsection{Quasi-blocky RC}

As it follows from figures 3 and 4 a quasi-blocky RC has a better capability of adsorbing onto a quasiblocky RS rather than onto a quasi-alternating RS. As $\beta$ increases, the tuning of the character of correlations in both RC and RS occurs, figure 5(a), (d): When $c_{\mathrm{s}}=0.5\left(=c_{\mathrm{p}}\right)$, both $\lambda_{\mathrm{p}}$ and $\lambda_{\mathrm{s}}$ slightly increase whereas at $c_{\mathrm{s}}=-0.5\left(=-c_{\mathrm{p}}\right)$, both $\lambda_{\mathrm{p}}$ and $\lambda_{\mathrm{s}}$ move towards each other, i.e., the positive $\lambda_{\mathrm{p}}$ decreases and the negative $\lambda_{\mathrm{s}}$ increases. At $c_{\mathrm{s}}=0$, an interesting behavior is observed: the RC cluster parameter does not change but the RS cluster parameter increases. At $c_{\mathrm{s}}=-1$, the surface regularly alternates (i.e., quenches) which cannot change "by definition", and the RC should adjust itself; hence, $\lambda_{\mathrm{s}}$ decreases and becomes negative.

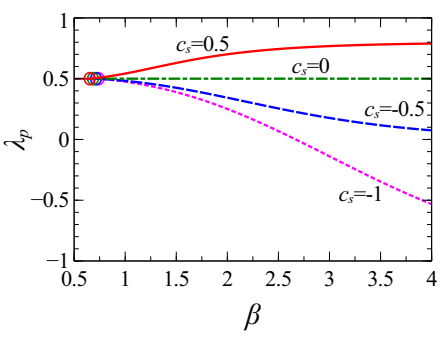

(a)

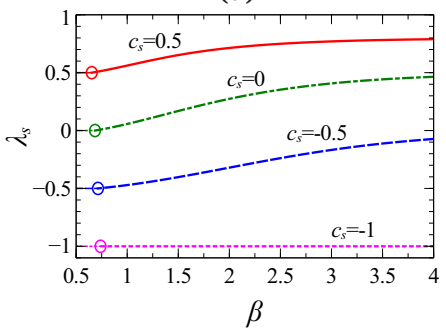

(d)

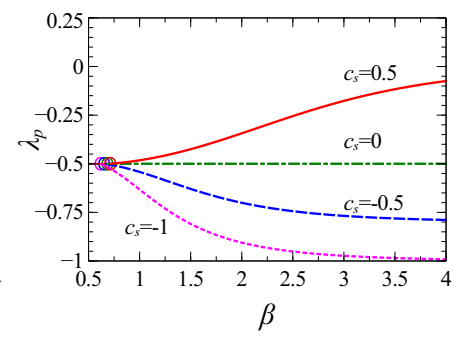

(b)

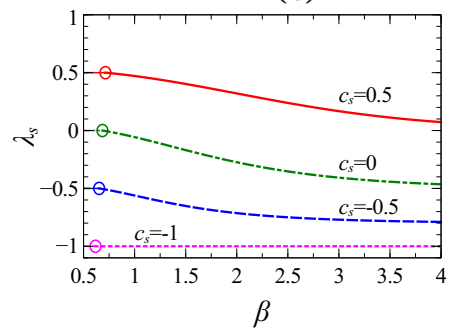

(e)

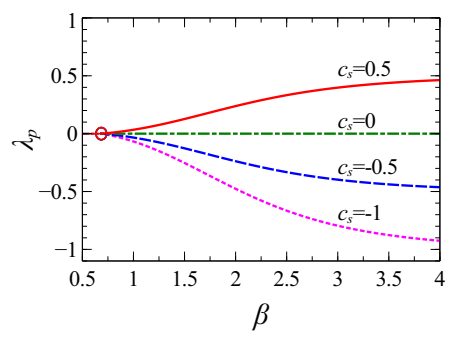

(c)

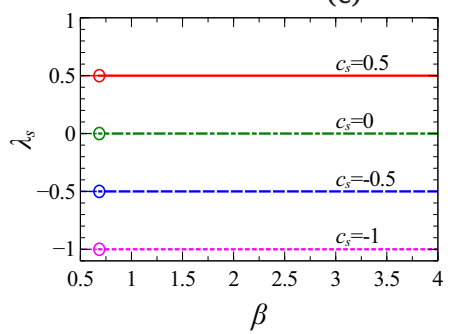

(f)

Figure 5. (Color online) Cluster parameters for RC (a)-(c) and RS (d)-(f) as functions of inverse temperature $\beta$ calculated for $f_{A}=g_{\mathrm{a}}=0.5, c_{\mathrm{p}}=0.5(\mathrm{a}, \mathrm{d}),-0.5(\mathrm{~b}, \mathrm{e}), 0(\mathrm{c}, \mathrm{f})$ and various $c_{\mathrm{s}}$ as indicated.

\subsubsection{Quasi-alternating RC}

Here, the same tendency to tuning as in the previous case is observed, figure 5 (c), (f). Hence, all the corresponding arguments may be straightforwardly reproduced with "the change of the sign": at 
$c_{\mathrm{s}}=-0.5\left(=c_{\mathrm{p}}\right)$ both $\lambda_{\mathrm{p}}$ and $\lambda_{\mathrm{s}}$ slightly decrease, at $c_{\mathrm{s}}=0.5\left(=-c_{\mathrm{p}}\right) \lambda_{\mathrm{p}}$ and $\lambda_{\mathrm{s}}$ move towards each other, at $c_{\mathrm{s}}=0 \lambda_{\mathrm{p}}$ does not change and $\lambda_{\mathrm{s}}$ decreases, at $c_{\mathrm{s}}=-1, \lambda_{\mathrm{p}}$ decreases towards -1 .

\subsubsection{Bernoullian RC}

With respect to the transition point and the adsorbed amount, figures 3 and 4 the behavior of the annealed Bernoullian copolymer does not depend on the correlations in the annealed RS (the latter can be even quenched regularly alternating with $c_{\mathrm{S}}=-1$ ). For the second moments of monomers and sites distributions in the RC and RS, respectively, the following regularity is observed: the a posteriori cluster parameters $\lambda_{\mathrm{s}}$ in the RS - the "partner" of the Bernoullian RC — does not change with $\beta$ and remains equal to the a priori correlation parameter $c_{\mathrm{s}}$, figure 5(b), (e). The "double Bernoullian" case $c_{\mathrm{p}}=c_{\mathrm{s}}=0$ is remarkable: the second moment does not change both in RC and RS.

\subsubsection{Summary for the symmetric case}

The analysis of the first and the second moments of monomers and site distributions in RC and RS, respectively, in the symmetric case shows that upon a decrease in temperature (increase in $\beta$ ), the increase in the number of good contacts and the corresponding decrease in the number of bad contacts is implemented via rearrangement of $A$ and $B$ monomer units ( $a$ and $b$ sites) in the RC chain (on the RS). We have also seen that the first moments (and, in some cases, the second moments) keep their expected values and, in this sense, the annealed approximation turns out to be more accurate than in the case of the (more simple) reference system. This also means that an improvement of the annealed approximation with the aid of the first-order Morita approximation (and the second order-in the case of the Bernoullian RC and RS) will produce no effect because the corresponding Morita constraints are already satisfied in the annealed system and the same results will be obtained.

\subsection{The effect of asymmetry}

As we have seen, in the symmetric case, the adsorption of annealed RC onto annealed RS exhibits the most interesting features, the most essential among them being the correct (with respect to the $a$ priori probabilities) values of the first moments of monomers and the distributions of sites meaning that the overall composition of the RC and RS does not change during the interaction. This is the consequence of the system symmetry. When both the RC and the RS are, in addition, Bernoullian, the second moments keep their correct values too.

Let us now analyze how a violation of the system's symmetry affects the RC adsorption onto the RS. In principle, the symmetry can be destroyed in two different ways: one can either (1) choose non-equal probabilities for $A$ and $B$ units ( $a$ and $b$ sites) to appear in the RC sequence (on the RS), i.e., set $f_{A}, g_{\mathrm{a}} \neq 0.5$ or (2) change the value of one of the interaction parameters $\epsilon_{i j}$. To see how different ways of introducing the asymmetry influence the adsorption, let us take the (simplest) set of parameters with $f_{A}=g_{\mathrm{a}}=0.5$, $c_{\mathrm{p}}=c_{\mathrm{s}}=0$ (both RC and RS are Bernoullian), $\epsilon_{A a}=\epsilon_{B b}=-1$, and $\epsilon_{A b}=\epsilon_{B a}=0$ as the reference. We will change one of the composition or the interaction parameters, keeping other parameters unchanged, as in the reference system. In particular, we (1) slightly change the probabilities of the appearance of $A$ and $B$ monomers in the RC, $f_{A}=0.6$, keeping for the RS $g_{\mathrm{a}}=0.5$ or (2) increase the attraction of $A$ monomer to a site by setting $\epsilon_{A a}=-1.2$ or (3) make one of the bad contacts repulsive instead of neutral by setting $\epsilon_{A b}=+1$, or (4) turn the good $B b$ contacts into bad by setting $\epsilon_{B b}=\epsilon_{A b}=\epsilon_{B a}=0$.

These four cases are compared in figure 6 The results of the comparison are as follows: (1) an increase of the probability of $A$ monomers present in the sequence, changes neither the position of the adsorption transition point nor the shape of the temperature dependence of the adsorbed fraction (and of the GF smallest singularity). At the same time, the RC and the RS compositions behave differently with the change of temperature: as the inverse temperature $\beta$ increases, composition of the RC remains the same whereas the fraction of $a$-sites increases and tends to 0.6. (2) An increase in the $A a$ affinity results in the shift of the transition point toward smaller $\beta$, while with an increase of $\beta$, the (expected) growth is observed in the amount of $A$ monomers and $a$ sites providing the most favorable $A a$ and $B b$ contacts. (3) An increase in the $A b$ interaction energy, shifts the transition point to larger values of $\beta$; fractions of $A$ monomers 


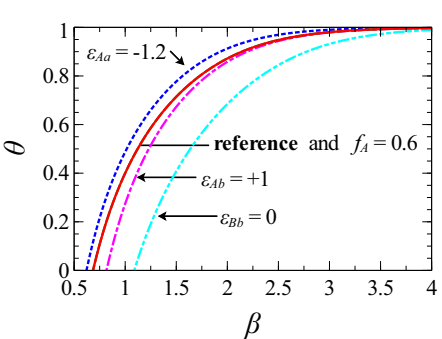

(a)

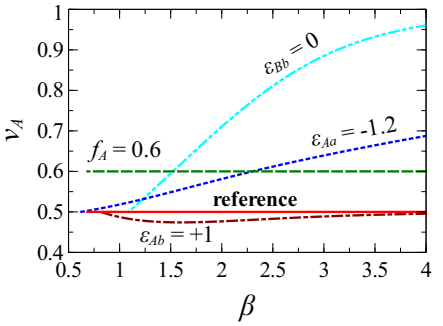

(b)

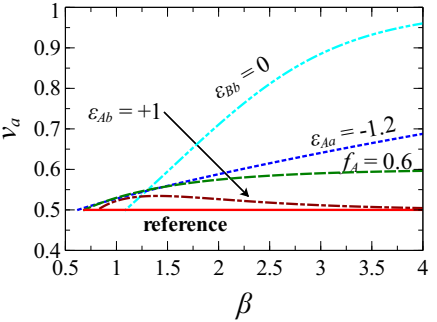

(c)

Figure 6. (Color online) Overall adsorbed fraction (a), fraction of $A$-monomers (b) and $a$-sites (b) as functions of inverse temperature $\beta$ calculated for symmetric reference system (red solid curves) with $f_{A}=g_{\mathrm{a}}=0.5, c_{\mathrm{p}}=c_{\mathrm{S}}=0, \epsilon_{A a}=\epsilon_{B b}=-1$, and $\epsilon_{A b}=\epsilon_{B a}=0$. Other curves are for the systems differing from the reference one by one of the parameters, indicated at the curve.

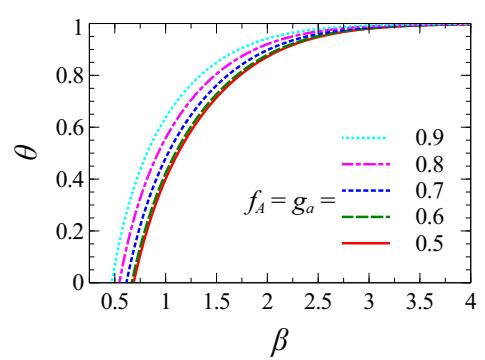

(a)

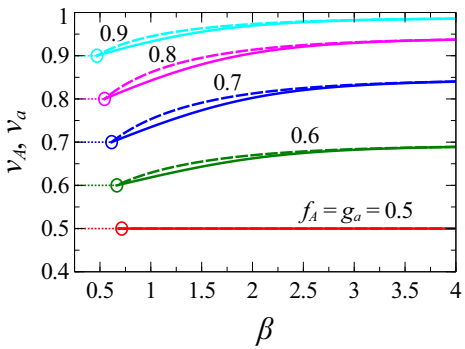

(b)

Figure 7. (Color online) Overall adsorbed fraction (a) and fractions of $A$-monomers (solid curves) and $a$ sites (dashed curves) (b) as functions of inverse temperature $\beta$ calculated for $c_{\mathrm{p}}=c_{\mathrm{s}}=0, \epsilon_{A a}=\epsilon_{B b}=-1$, $\epsilon_{A b}=\epsilon_{B a}=0$, and equal fractions of $A$-monomers and $a$-sites $\left(f_{A}=g_{\mathrm{a}}\right)$ as indicated.

and $B$ sites slightly decrease just after the adsorption transition; as $\beta$ increases, they both tend to 0.5 , in order to have a maximum of possible favorable $A a$ and $B b$ contacts. (4) The transformation of attractive $B b$ contacts intro neutral ones shifts the transition point towards larger $\beta$ and leads to massive $B \rightarrow A$ and $b \rightarrow a$ transformations with an increasing $\beta$.

The observed behavior is in agreement with the behavior of the reference system (RC adsorbing onto a homogeneous surface, see section 3), where the equilibrium is shifted towards transformation of nonadsorbing monomer units into adsorbing ones. In our case, we encounter more complicated coherent monomers and transformations of sites. As soon as some preferences appear in the transformation reaction constants (governed by $f_{A}$ and $g_{a}$ ) or in the interaction map, the equilibrium shifts toward these preferences.

There is another interesting way to partly violate the symmetry of the system. By comparing the fraction of $A$-monomers with that of $a$-sites and the fraction of $B$-monomers with that of $b$-sites we can obtain the upper boundary for the maximum possible fraction of good contacts. Since good contacts are the $A a$ and the $B b$ ones, then $\theta_{\text {good }}=\theta_{A a}+\theta_{B b}$. Obviously, $\theta_{A a} \leqslant \min \left(f_{A}, g_{a}\right), \theta_{B b} \leqslant \min \left(f_{B}, g_{b}\right)$, hence, $\theta_{\text {good }}^{\max }=\min \left(f_{A}, g_{\mathrm{a}}\right)+\min \left(f_{B}, g_{b}\right)$. In the symmetric case, $f_{A}=f_{B}=g_{\mathrm{a}}=g_{b}=0.5$, therefore, $\theta_{\text {good }}^{\max }=1$. If we choose the RC and the RS compositions, so that $f_{A}=g_{\mathrm{a}}$ and $f_{B}=g_{b}$, the upper boundary for the total fraction of good contacts will still be equal to one, as it is in the symmetric case.

Temperature dependences of the overall adsorbed fraction, RC and RS compositions and cluster parameters for $f_{A}=g_{\mathrm{a}} \geqslant 0.5$ and $c_{\mathrm{p}}=c_{\mathrm{s}}=0$ are presented in figure 7 We see that in spite of the invariance of $\theta_{\text {good }}^{\max }$, a simultaneous increase in the fraction of $A$-monomers and $a$-sites favors adsorption: the transition point shifts to the lower $\beta$ values, for a given temperature, the total adsorbed fraction is larger in the case of larger $f_{A}=g_{\mathrm{a}}$. Moreover, with an increasing $\beta$, the fractions of the major $A$ and $a$ components grow and the fractions of the minor $B$ and $b$ components correspondingly decrease. It is also clear that if we take $f_{A}=g_{\mathrm{a}}<0.5$, the picture will remain qualitatively and quantitatively the same with respect to 
major and minor components (here, $B$ and $b$ are major components, $A$ and $a$ are minor components). The only exception is the symmetric case $f_{A}=g_{\mathrm{a}}=0.5$, where there is a strict $A / B$ and $a / b$ balance. That is, one can say that the equilibrium gets shifted towards the major component, and the situation becomes similar in some sense to that in the reference system of section 3

\section{Conclusion}

We have considered a two-dimensional partially directed walk (2D-PDW) model of a two-letter $(A B)$ random copolymer (RC) adsorption onto a two-letter $(a b)$ random surface (RS). This model was introduced in our previous work [31] where it was treated by using the combination of the annealed approximation (to perform double averaging over the sequence and surface disorder) with the generating functions (GFs) approach (to sum over polymer conformations). In contrast to [31], in the present work we have gone beyond the transition point and studied the temperature dependences of various observables for the annealed symmetric system. This choice was motivated for several reasons: The annealed approximation provides a zero-order rough approximation to a realistic quenched system; on the other hand, it can serve as a prototype of real physical systems like two-state polymers [39]. The system was chosen to be "twofold” symmetric: with respect to the composition of the RC and RS and with respect to the interaction map (good attractive $A a$ and $B b$ contacts had the same energy, while both bad $A b$ and $B a$ contacts were equally neutral). In [31] it was shown that in this case the system has the most interesting phase diagram. Finally, the symmetry of the system makes highly unpredictable interconversion of monomers and sites ( $A \rightleftharpoons B$ and $a \rightleftharpoons b$ ) in the annealed system. Therefore, special attention was paid to a posteriori moments of distributions of monomers and sites.

We have shown that in the considered symmetric case, the expected a posteriori compositions of the $\mathrm{RC}$ and the RS correspond to the a priori probabilities to meet $A$ monomers and a sites in the RC monomer sequence / RS site sequence and do not change with the temperature. At the same time, the a posteriori cluster parameter (related to the second moments of distributions of monomers and sites) in the RC and RS changes with the temperature, indicating that monomers and sites rearrange in the RC and the RS to provide a better matching between them and, hence, a stronger adsorption. A special case is the one where both the RC and the RS are Bernoullian: in this situation, both first and second moments keep their correct values at any temperature.

We have also studied the effect of the system symmetry violation on the adsorption behavior. There are various ways of doing this and all of them shift the equilibrium towards the major component and/or more favorable contacts.

\section{Acknowledgements}

The author is grateful to Dr. Oleg V. Borisov for his critical comments and suggestions. This work was partially supported by the Government of the Russian Federation, grant 074-U01.

\section{References}

1. Privman V., Švrakić N.M., Directerd Models of Polymers, Interfaces, and Clusters: Scaling and Finite-Size Properties, Lecture Notes in Physics, Springer-Verlag, Berlin Heidelberg, 1989.

2. Janse Van Rensburg E.J., The Statistical Mechanics of Interacting Walks, Polygons, Animals and Vesicles, Oxford Lecture Series in Mathematics and Its Applications, Oxford University Press, 2000.

3. Janse van Rensburg E.J., J. Phys. A: Math. Gen., 2003, 36, No. 15, R11; doi 10.1088/0305-4470/36/15/201

4. Privman V., Forgacs G., Frisch H.L., Phys. Rev. B, 1988, 37, 9897; doi 10.1103/PhysRevB.37.9897

5. Carvalho M.C.T.P., Privman V., J. Phys. A: Math. Gen., 1988, 21, No. 21, L1033; doi 10.1088/0305-4470/21/21/007

6. Forgacs G., Privman V., Frisch H.L., J. Chem. Phys., 1989, 90, No. 6, 3339; doi 10.1063/1.455888

7. Brak R., Owczarek A.L., Rechnitzer A., Whittington S.G., J. Phys. A: Math. Gen., 2005, 38, No. 20, 4309; doi $10.1088 / 0305-4470 / 38 / 20 / 001$

8. Brak R., Iliev G.K., Rechnitzer A., Whittington S.G., J. Phys. A: Math. Theor., 2007, 40, No. 17, 4415; doi $10.1088 / 1751-8113 / 40 / 17 / 001$ 
9. Owczarek A.L., Prellberg T., Rechnitzer A., J. Phys. A: Math. Theor., 2008, 41, No. 3, 035002; doi $10.1088 / 1751-8113 / 41 / 3 / 035002$.

10. Owczarek A.L., J. Stat. Mech., 2009, 2009, No. 11, P11002; doi 10.1088/1742-5468/2009/11/P11002

11. Brak R., Iliev G.K., Owczarek A.L., Whittington S.G., J. Phys. A: Math. Theor., 2010, 43, No. 13, 135001; doi 10.1088/1751-8113/43/13/135001

12. Iliev G.K., Janse van Rensburg E.J., J. Stat. Mech., 2011, 2011, No. 11, P11013; doi 10.1088/1742-5468/2011/11/P11013

13. Brak R., Guttmann A.J., Whittington S.G., J. Phys. A: Math. Gen., 1992, 25, No. 9, 2437; doi 10.1088/0305-4470/25/9/017

14. Owczarek A.L., Prellberg T., Brak R., J. Stat. Phys., 1993, 72, No. 3-4, 737; doi 10.1007/BF01048031

15. Zhou H., Zhou J., Ou-Yang Z.C., Kumar S., Phys. Rev. Lett., 2006, 97, 158302; doi 10.1103/PhysRevLett.97.158302

16. Owczarek A.L., Prellberg T., J. Stat. Mech., 2007, 2007, No. 11, P11010; doi 10.1088/1742-5468/2007/11/P11010

17. Nguyen G.B., Pétrélis N., J. Stat. Phys., 2013, 151, No. 6, 1099; doi 10.1007/s10955-013-0748-2

18. Orlandini E., Whittington S.G., J. Phys. A: Math. Gen., 2004, 37, No. 20, 5305; doi 10.1088/0305-4470/37/20/004

19. Orlandini E., Tesi M.C., Whittington S.G., J. Phys. A: Math. Gen., 2004, 37, No. 5, 1535; doi $10.1088 / 0305-4470 / 37 / 5 / 005$.

20. Orlandini E., Tesi M.C., J. Math. Chem., 2009, 45, No. 1, 72; doi 10.1007/s10910-008-9369-4

21. Iliev G.K., Orlandini E., Whittington S.G., J. Phys. A: Math. Theor., 2010, 43, No. 31, 315202; doi 10.1088/1751-8113/43/31/315202

22. Orlandini E., Whittington S.G., J. Phys. A: Math. Theor., 2010, 43, No. 48, 485005; doi 10.1088/1751-8113/43/48/485005

23. Osborn J., Prellberg T., J. Stat. Mech., 2010, 2010, No. 09, P09018; doi 10.1088/1742-5468/2010/09/P09018

24. Owczarek A.L., J. Phys. A: Math. Theor., 2010, 43, No. 22, 225002; doi $10.1088 / 1751-8113 / 43 / 22 / 225002$

25. Tabbara R., Owczarek A.L., J. Phys. A: Math. Theor., 2012, 45, No. 43, 435002; doi 10.1088/1751-8113/45/43/435002

26. Iliev G.K., Whittington S.G., J. Phys. A: Math. Theor., 2012, 45, No. 18, 185003; doi 10.1088/1751-8113/45/18/185003

27. Brak R., Dyke P., Lee J., Owczarek A.L., Prellberg T., Rechnitzer A., Whittington S.G., J. Phys. A: Math. Theor., 2009, 42, No. 8, 085001; doi 10.1088/1751-8113/42/8/085001

28. Lam P.M., Zhen Y., J. Stat. Mech., 2010, 2010, No. 05, P05011; doi 10.1088/1742-5468/2010/05/P05011

29. Orlandini E., Rechnitzer A., Whittington S.G., J. Phys. A: Math. Gen., 2002, 35, 7729; doi 10.1088/0305-4470/35/36/303

30. Soteros C.E., Whittington S.G., J. Phys. A: Math. Gen., 2004, 37, R279; doi 10.1088/0305-4470/37/41/R01.

31. Polotsky A.A., J. Phys. A: Math. Theor., 2012, 45, No. 42, 425004; doi $10.1088 / 1751-8113 / 45 / 42 / 425004$

32. Whittington S.G., J. Phys. A: Math. Gen., 1998, 31, No. 44, 8797; doi 10.1088/0305-4470/31/44/008

33. Iliev G., Orlandini E., Whittington S.G., J. Phys. A: Math. Theor, 2011, 44, 405004; doi 10.1088/1751-8113/44/40/405004

34. Polotsky A.A., J. Phys. A: Math. Theor., 2014, 47, No. 24, 245002; doi 10.1088/1751-8113/47/24/245002

35. Iliev G., Orlandini E., Whittington S.G., Eur. Phys. J. B, 2004, 40, No. 1, 63; doi 10.1140/epjb/e2004-00239-1

36. Iliev G.K., Janse van Rensburg E.J., J. Stat. Mech., 2012, 2012, No. 01, P01019; doi 10.1088/1742-5468/2012/01/P01019

37. Iliev G.K., Whittington S.G., Phys. Rev. E, 2013, 88, 052105; doi 10.1103/PhysRevE.88.052105

38. Grosberg A.Y., J. Stat. Phys., 1985, 38, No. 1-2, 149; doi 10.1007/BF01017854

39. Yoshinaga N., Kats E., Halperin A., Macromolecules, 2008, 41, No. 20, 7744; doi 10.1021/ma801199j

40. Currie E.P.K., Cohen Stuart M.A., Borisov O.V., Macromolecules, 2001, 34, No. 4, 1018; doi 10.1021/ma991540n

41. Polotsky A.A., Degenhard A., Schmid F., J. Chem. Phys., 2009, 131, 054903; doi 10.1063/1.3193723

42. Ziebarth J.D., Williams J., Wang Y., Macromolecules, 2008, 41, No. 13, 4929; doi 10.1021/ma800212n

43. Dill K.A., Biochemistry, 1985, 24, No. 6, 1501; doi 10.1021/bi00327a032

44. Lau K.F., Dill K.A., Macromolecules, 1989, 22, No. 10, 3986; doi 10.1021/ma00200a030

45. Birshtein T.M., Macromolecules, 1979, 12, No. 4, 715; doi 10.1021/ma60070a036 


\title{
Адсорбція симетричного випадкового кополімера на симетричну випадкову поверхню: відпалений випадок
}

\author{
А.А. Полоцький \\ 1 Інститут макромолекулярних сполук, Російська академія наук, 199004 Санкт-Петербург, Російська \\ Федерація \\ 2 Санкт-Петербурзький національний дослідницький університет інформаційних технологій, механіки та \\ оптики (університет ITMO), 197101 Санкт-Петербург, Російська Федерація
}

\begin{abstract}
Адсорбція симетричного $(A B)$ випадкового кополімера (BK) на симетричну $(a b)$ випадкову неоднорідну поверхню (ВП) вивчається у наближенні відпалу із використаннями для полімера двовимірної моделі частково напрямлених блукань. Показано, що у симетричному випадку очікувані а posteriori концентрації ВК і ВП мають правильні значення (які відповідають їх a priori ймовірностям) і не змінюються, в залежності від температури, в той час, як другі моменти розподілів мономерів і вузлів в ВК і ВП змінюються. Це показує, що мономери і вузли взаємно не перетворюються, а лише перегруповуються, щоб забезпечити краще допасовування між ними і, як результат, сильнішу адсорбцію ВК на ВП. Проте будь-яке порушення симетрії системи зсуває рівновагу у напрямку основної компоненти і/або до більш сприятливих контактів та приводить до взаємоперетворення мономерів і вузлів.
\end{abstract}

Ключові слова: випадковий кополімер, випадкова поверхня, полімерна адсорбція, наближення відпалу, генеруючі функції 


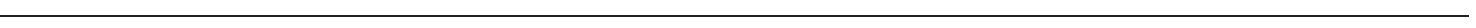

